\title{
Research on Reverse Engineering Technology of Laser Cladding Repairing Gears
}

\author{
Shi Juan \\ Logistics Engineering College \\ Shanghai Maritime University \\ Shanghai, China, 13661499048 \\ shijuan@shmtu.edu.cne
}

\author{
Bai Shunqin \\ R\&D \\ Magneti Marelli Automotive Lighting Co.,Ltd \\ Shanghai, China, 15216663328 \\ baishunqin@126.com
}

\begin{abstract}
Taking the spur gear as the study object, this paper introduces the method of reconstruction wear gear model by reverse engineering for gear laser cladding repairing. Using the laser scanning measuring system, through the reverse digital, point cloud pre-processing technology, matched 3D CAD model of the impaired standard involute gear. Through point cloud alignment theory and method of gear damage, made the alignment analysis for the CAD model before wear and after, and the quantitative analysis of wear layer is achieved. The method provides conditions for laser cladding repairing of wear gear.
\end{abstract}

Keywords-component; Reverse engineering; Surface fitting reconstruction; Alignment analysis; Repairing

\section{INTRODUCTION}

Tooth wear is one of the common forms of failure of the gears. Using the laser cladding technique to repair the wear tooth surface can extend the life of the gear and greatly reduce costs, especially for expensive big modulus gear and imported gear. Due to the uncertainty and complexity characteristics of the wear location and the amount of wear, cladding layer too thick or not enough always occur when repair damaged gear with laser cladding technique. The former situation will add to the final processing workload, the latter situation can't be up to repair effect. Reverse engineering is a technique which based on a high-speed digital scanner to accurately scan the existing model with high-speed, to obtain its three-dimensional contour data and realize surface reconstruction with the reverse software. Via reverse engineering we can obtain the accurate damaged location and the size of the damage amount. This makes accurate repair damaged gear become possible.

\section{ACQUISITION OF WEAR GEAR SURFACE POINT CLOUD}

Getting the surface point cloud data in high accuracy is the foundation and key of wear gear model reconstruction. Here use the scan measurement system of Creaform company to get wear gear surface point cloud data.

Due to the complex structural characteristics of gear surface, we use parameters as shown in the Table 1 to san in order to ensure the integrity and accuracy of the data. While using the scanning policy of the positioning point stitching, scan the upper and lower surfaces of the gear, then splice he independent scan data of these two surfaces with the positioning point by itself. This scanning strategy has the advantage in that it can delete repetitive portion of the scan data in the post-splicing process automatically fill the missing part to ensure accuracy, at the same time improve the scanning efficiency. Figure 1 is the scan picture of upper and lower surfaces of damaged gear.

TABLE I. THE LIST OF SCAN PARAMETERS

\begin{tabular}{|c|c|c|c|}
\hline $\begin{array}{c}\text { Laser power } \\
(\%)\end{array}$ & $\begin{array}{c}\text { Flash time } \\
(\mathbf{m s})\end{array}$ & $\begin{array}{c}\text { Scan domain } \\
(\mathbf{m m})\end{array}$ & $\begin{array}{c}\text { Scan resolution } \\
(\mathbf{m m})\end{array}$ \\
\hline 94 & 10.0 & $300 \times 300 \times 300$ & 0.59 \\
\hline
\end{tabular}

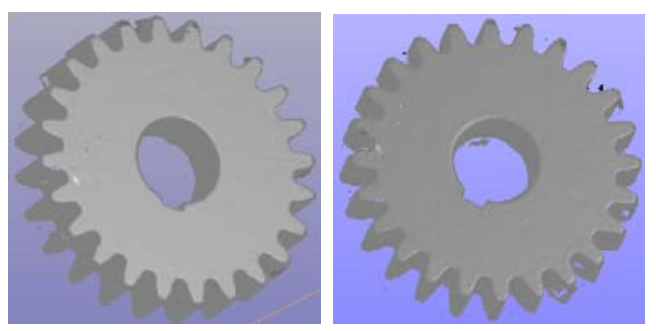

Figure 1. Scan picture of upper and lower surfaces of gear

\section{RECONSTRUCTION OF WEAR GEAR SOLID MODEL}

The typical fast surface reconstruction software Geomagic Studio was used to complete the processing of point cloud data. Geomagic reverse design principle is that many of the small space triangle approximation restore CAD solid model. In modeling strategy, it create NURBS surface model fitting with NURBS surface patches. The concrete surface reconstruction process is divided into point stage, the polygon phase and shape stage, they are contact with each other closely.

\section{A. Point stage}

Pre-processing of wear gear point cloud Includes the following technical operations: delete non-connected items, remove vitro solitary point, reduce noise, uniform sampling, package, manual registration, global registration and so on.

1) Remove the non-connection point cloud and in vitro solitary point, filter noise points. Due to the measurement accuracy of the equipment, scanning speed, and the operator's experience, a slight vibration of the scanning apparatus and the surface quality of the part being measured, it is inevitable to 
scan some rough, non-uniform point cloud and noise points in the process. These points should be cleared.

2) The point cloud data sampling. Reduce the point cloud data can make the data computing faster, improve the operation efficiency. Maintaining the accuracy of the model and sampling the data points to streamline the point cloud data amount. Figure 2 is gear up and down two surface sampling point clouds.

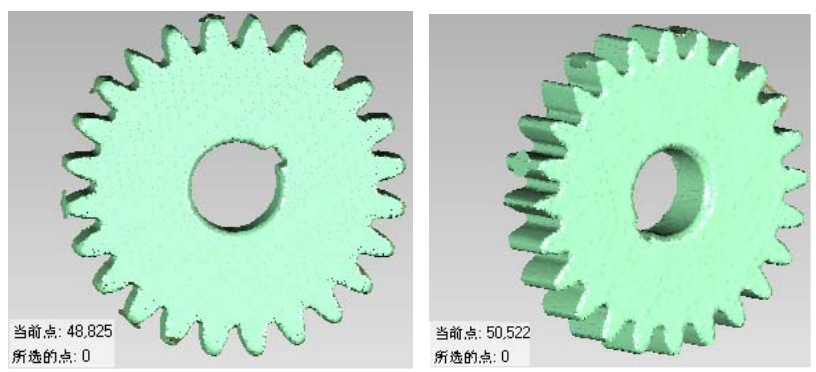

Figure 2. The gear surface sampling point cloud

3) Point cloud data stitching based on its own anchor. It is difficult to get a complete point cloud of gear because of its shaft bore and keyway structure, so we should splice the two surfaces of the upper and lower point cloud data based on common characteristic[1], the common characteristic are the anchor points on the gear. Find the common feature points in the fixed and floating windows of two cloud data, as shown in Figure 3, selecting the four positioning point of the tooth surface as alignment point, two cloud data will certain automatic alignment in the window underside of the working area. In order to further reduce the alignment error, it is possible through the "global Register" command to recalculation of scanning data.

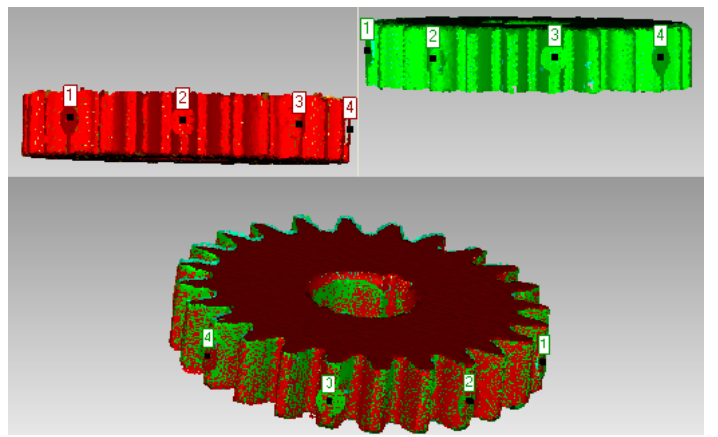

Figure 3. "Manually register" registration point selection diagram

Global registration is completed, it still the point cloud data. Use the "merge" command in order to achieve the merger of two or more point cloud data as a whole, and automatically perform point cloud noise reduction, unified sampling package and generate polygon model. The combined effect is shown in Figure 4.

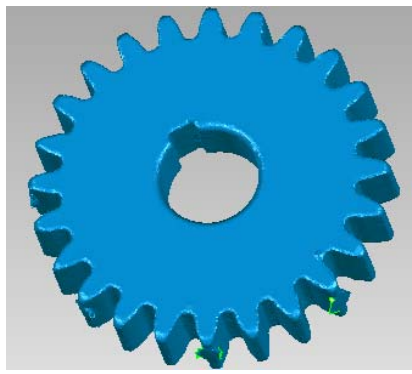

Figure 4. Merge effect diagram

\section{B. Polygon stage}

The polygons stage is a series of technical after processing point cloud data package to get a complete and ideal polygon data model, and lay the foundation for surface fitting. Its main process shown in Figure 5.

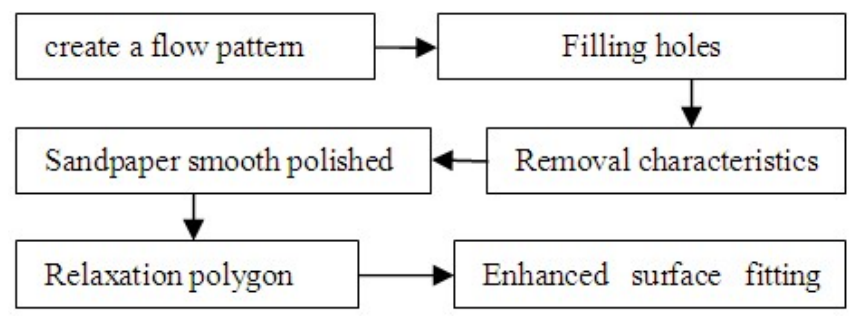

Figure 5. The polygons stage operation flowchart

\section{Shape stage}

This stage is a series of technical processing for the triangular mesh surface data get from polygon stage to get a ideal surface model. The processes are shown in Figure 6. After a series of processing, a gear model obtained which shown in Figure 7.

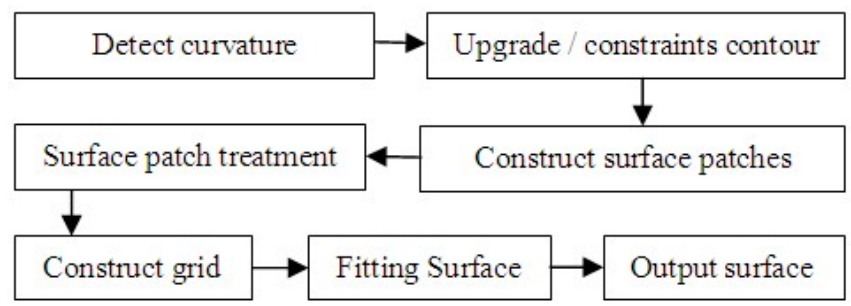

Figure 6. The surface fitting reconfigurable processing operation flowchart

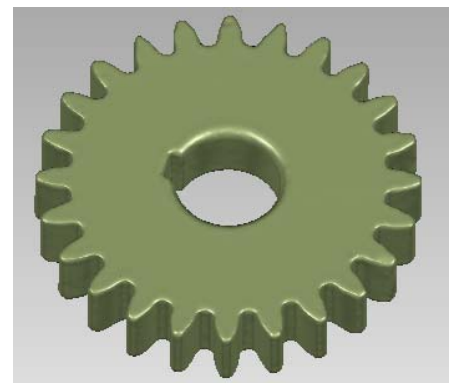

Figure 7. The gear surface fitting effect diagram 


\section{ALIGNMENT ANALYSIS OF GEAR WEAR PARTS AND STANDARD PARTS}

Import the digitized model of wear gear got from the previous step and the standard model in a three-dimensional analysis software Geomagic Qualify 11. And use the best fit alignment method to make a alignment registration, alignment effect is shown in Figure 8. The brown one is the standard model of involute straight tooth cylindrical wear gear part which as the reference model. The gray one is the wear gear digital model for the test model. The deviation of approximate location and extent between digital model and the standard model can be observed in the best-fit alignment state.

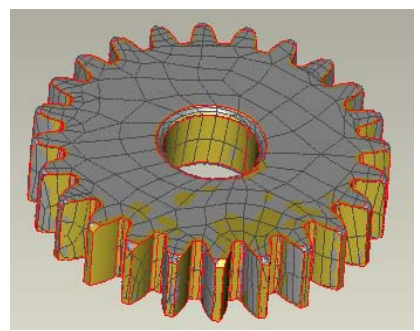

Figure 8. The alignment of the standard model and the digital model registration diagram

After "3D compare" function analysis, we can obtain the chromatography deviation map of two models as shown in the Figure 9, the deviation of most area are in the range of -0.160 to 0.160 , it mainly because of spraying the developer to gear before scanning. Tooth chamfering is not the structure to repair in this paper, so the precise size of the chamfer is not considered when drew standard CAD model. It makes a large positive deviation in tooth, it will not be considered. Obviously we can see two tooth surface which be man-made destruction have maximum deviation.

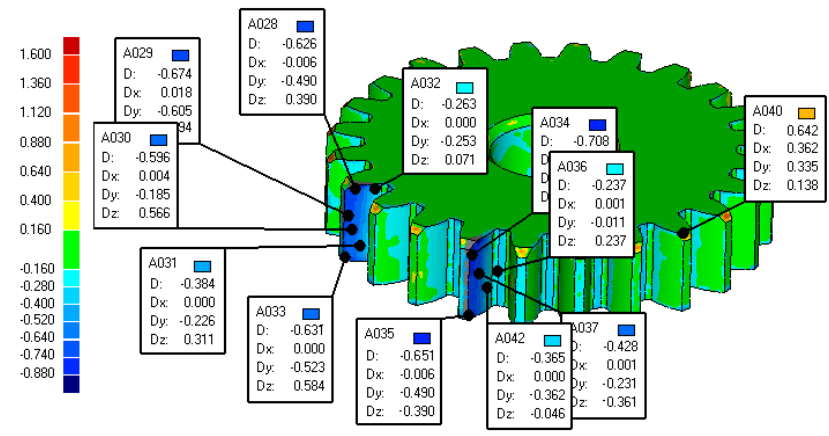

Figure 9. Contrast chromatograms and wear values mark between the standard model and the digital model

Create comments in the badly worn areas can get the Specific deviation marked value in this point. Place where the most serious damage is the addendum, the amount of wear is $0.708 \mathrm{~mm}$, the amount of wear gradually decreases from the addendum to the tooth root, the tooth root wear $0.237 \mathrm{~mm}$, the data obtained matched with the actual gear wear values, we can determine processes and parameters of laser cladding to repair the wear gear according the wear deviation .

\section{CONCLUSION}

In this paper, use the method of reverse engineering to get the point cloud of wear gear, then obtain the entity CAD model of wear gear after a series of processing. Get wear location coordinates and the data of wear amount after alignment analysis between it and its standard CAD model. And quantitative analysis of the amount of wear is achieved

\section{REFERENCES}

[1] Cheng siyuan, Xie shaowang. "Geomagic Studio reverse engineering technology and applications,” Tsinghua University Medical Press, 2010.

[2] Zhang changxue, Zhang xue , Gaoxuejun, "Reverse modeling technology and product innovation design”, Peking University Press, 2009.

[3] Xu bingshi, State of the art and future development in remanufacturing engineering. Transactions Ions of Materials and Heat Treatment, 2010, 31(1): 10-14.

[4] Chen jiang, Liu yulan, “The Engineering Application of Laser Remanufacturing Technology,” China surface Engineering, 2006, 19(5): 50-55. 